\title{
Analytic Fields on Riemann Surfaces. II
}

\author{
V.G. Knizhnik \\ Landau Institute for Theoretical Physics, Moscow, USSR
}

\begin{abstract}
The properties of analytic fields on a Riemann surface represented by a branch covering of $\mathbb{C P}^{1}$ are investigated in detail. Branch points are shown to correspond to the vertex operators with simple conformal properties. As applications we compute determinants of $\bar{\partial}_{j}$ operators for $Z_{n}$-symmetric surfaces and obtain various representations for the two-loop measure in the bosonic string theory together with various identities for theta-functions of hyperelliptic surfaces. We also present an integral representation for the quantum part of the twist field correlation functions, which describe propagation of the string on the orbifold background. We also calculate the quantum part of the structure constants of the twist-field operator algebra, generalizing the results of Dixon, Friedan, Martinec, and Shenker.
\end{abstract}

\section{Introduction}

The chiral pairs of anticommuting fields in two dimensions are known to play an important role in string theory and in conformal quantum field theory. Recently it was shown $[1,2]$ that the conformal theory of such fields can be constructed on an arbitrary Riemann surface with the special singular metric, under a careful account of all anomalies and zero modes. In the present paper we will continue the study of analytic fields, but now it will be more convenient for us to represent the surface as a branched covering of $\mathbb{C P}^{1}$.

Let us denote by $Z$ the covering map of a surface $X$ on $\mathbb{C P}^{1}$ :

$$
Z: X \rightarrow \mathbb{C P}^{1},
$$

and choose the metric on $X$ to be

$$
g_{z z}=g_{\bar{z} \bar{z}}=0, \quad g_{z \bar{z}}=1
$$

the complex structure of $X$ being induced from $\mathbb{C P}^{1}$. 
We shall show that under such a choice each branch point $a_{i}$ corresponds to the primary conformal field $V_{i}\left(a_{i}\right)$ with very simple properties. Actually, the addition of $V_{i}$-operators to the correlation functions of fields on $\mathbb{C P}^{1}$ transforms them into the correlation functions of fields on $X$. Besides, various determinants are given by the correlation functions of $V_{i}$-operators, and we compute them in some special cases.

In Sect. 2 we introduce the fields $V_{i}\left(a_{i}\right)$ and find their conformal properties. Further, in Sect. 3 we study in detail Riemann surfaces with $Z_{n}$-symmetry, defined by the equation

$$
y^{n}=\left(Z-a_{1}\right) \ldots\left(Z-a_{m n}\right)
$$

in $\mathbb{C}^{2}=(y, Z)$. For such surfaces the correlation functions of $V_{i}\left(a_{i}\right)$ are particularly simple.

The important case of hyperelliptic surfaces $(n=2)$ is considered in Sects. 4-6. In Sect. 4 we apply our approach to derive an integral representation, analogous to the one used in minimal conformal models [3], for the quantum part of the correlation functions of $Z_{2}$-twists, which arise when one describes the string propagation on the $Z_{2}$ orbifold. This representation is generalized in Sect. 7 to $Z_{N}$-twists and appears to be particularly useful for the calculation of the structure constants of twist-field operator algebra and the four-point correlation functions.

In Sect. 5 we establish connection of our formulas with the theory of theta functions on hyperelliptic surfaces, and in Sect. 6 we find a simple expression for the two-loop vacuum amplitude in the model of closed oriented bosonic strings (ESVM).

From the very beginning we have to make a reservation that the impulse for the present research was given by the paper by Alexey Zamolodchikov on correlation functions of spin-fields in the Ashkin-Teller model [4]. Later some of his results were independently obtained by Dixon et al. [5]. The latter paper was also very important for us and the construction of Sect. 7 is strongly based on the results of that paper.

\section{Branch Points as Primary Conformal Fields}

In this section we consider the behaviour of quantum analytic fields on an arbitrary Riemann surface $X$ in a small vicinity $V$ of a branch point of the order $n$. We choose the complex single valued coordinate $y$ on $V$ in such a way that the covering map (1.1) on $V$ takes the form:

$$
Z(y)=a+y^{n},
$$

where $a$ is the coordinate of the branch point on $\mathbb{C P}^{1}$. We numerate $n$ consecutive sheets of the Riemann surface $X$ of the inverse map

$$
y(Z)=(Z-a)^{1 / n}
$$

by numbers $0,1, \ldots, n-1$.

Thus, when a point is moved around $Z=a$, its inverse image move from the $\ell$-th sheet to the $\ell+1(n-1+1 \equiv 0)$. The operation of such analytic continuation will be denoted by $\hat{\pi}_{a}$. On each sheet $\ell$ we consider a pair of analytic anticommuting fields $f^{(\ell)}$ and $\phi^{(\ell)}$ with integer spins $j$ and $(1-j)$ and with the action

$$
S^{(\ell)}=\int f^{(\ell)} \bar{\partial} \phi^{(\ell)} d^{2} Z .
$$


Here $\partial \equiv \partial / \partial Z$ (also $\bar{\partial} \equiv \partial / \partial \bar{Z}$ ) and $f^{(\ell)}(Z, \bar{Z})$ denotes the $f$-field at a point $y(Z)$ on the $\ell$-th sheet of the surface $X$.

We remind that the stress-energy tensor of the fields $f^{(\ell)}, \phi^{(\ell)}$ has the form

$$
T^{(\ell)}=-j f^{(\ell)} \partial \phi^{(\ell)}+(j-1) \phi^{(\ell)} \partial f^{(\ell)},
$$

and under conformal transformations

$$
Z \rightarrow \alpha(Z), \quad \bar{Z} \rightarrow \overline{\alpha(Z)}
$$

these fields transform as differentials:

$$
\begin{gathered}
f^{(\ell)}(\alpha, \bar{\alpha})(d \alpha / d Z)^{j}=f^{(\ell)}(Z, \bar{Z}), \\
\phi^{(\ell)}(\alpha, \bar{\alpha})(d \alpha / d Z)^{1-j}=\phi^{(\ell)}(Z, \bar{Z}) .
\end{gathered}
$$

In (2.4) the following normalization is assumed

$$
f^{(\ell)}\left(Z^{\prime}\right) \phi^{(\ell)}(Z) \simeq I\left(Z^{\prime}-Z\right)^{-1}+\text { Reg. terms },
$$

where $I$ denotes the identity operator.

When we move around the branch point we pass from one sheet of the surface $X$ to another. This implies the following boundary conditions to be satisfied ${ }^{1}$ :

$$
\begin{aligned}
& \hat{\pi}_{a} f^{(\ell)}(Z)=f^{(\ell+1)}(Z), \\
& \hat{\pi}_{a} \phi^{(\ell)}(Z)=\phi^{(\ell+1)}(Z) .
\end{aligned}
$$

To find out the behaviour of the fields $f^{(\ell)}, \phi^{(\ell)}, \ell=0, \ldots, n-1$ in the vicinity of the branch point it is useful to choose another basis diagonalizing $\hat{\pi}_{a}$ :

$k=0,1, \ldots, n-1$.

$$
\begin{aligned}
f_{k} & =\sum_{\ell=0}^{n-1} e^{-2 \pi i(k+j(1-n)) l / n} f^{(\ell)} \\
\phi_{k} & =\sum_{\ell=0}^{n=1} e^{2 \pi i(k+j(1-n)) l / n} \phi^{(\ell)}
\end{aligned}
$$

Here we have shifted $k$ by $j(1-n)$ for reasons which will become clear below.

From (2.9) it follows that

$$
\begin{aligned}
\hat{\pi}_{a} f_{k} & =e^{2 \pi i(k+j(1-n)) / n} f_{k}, \\
\hat{\pi}_{a} \phi_{k} & =e^{-2 \pi i(k+j(1-n)) / n} \phi_{k} .
\end{aligned}
$$

An important consequence of (2.9) is that the currents

$$
J_{k}=: f_{k} \phi_{k}:, \quad \bar{\partial} J_{k}=0
$$

are singlevalued functions of $Z$ in the vicinity of the branch point $a$.

In the remaining part of this section we shall show that with respect to the currents $J_{k}$ the branch point has the charge

$$
q_{k}=\frac{k+j(1-n)}{n}
$$

\footnotetext{
${ }^{1}$ We shall usually suppress the argument $\bar{Z}$ of $f$, $\phi$-fields because all their correlation functions do not depend on $\bar{Z}$ due to the equations of motion
} 
which implies that the current $J_{k}(Z)$ has the first order pole at $Z=a^{2}$ with the residue $q_{k}$ :

$$
J_{k}(Z)=q_{k}(Z-a)^{-1}+\text { Reg. terms } .
$$

The above relations are solved by expressing operators $f_{k}$, $\phi_{k}$ through $n$ analytic bosonic fields $\varphi_{k}, k=0, \ldots, n-1$ normalized as

$$
\left\langle\varphi_{k}(Z) \varphi_{m}\left(Z^{\prime}\right)\right\rangle=-\delta_{k m} \log \left(Z-Z^{\prime}\right),
$$

using the following bosonization rules:

$$
\begin{aligned}
& f_{k}=: e^{i \varphi_{k}}: \quad \phi_{k}=: e^{-i \varphi_{k}}: \\
& J_{k}=i \partial \varphi_{k} \\
& T_{k}=-j f_{k} \partial \phi_{k}+(1-j) \phi_{k} \partial f_{k}=1 / 2: J_{k}^{k}:+(1 / 2-j) \partial J_{k} .
\end{aligned}
$$

In terms of the fields $\varphi_{k}$ the relations (2.12), (2.13) require the following vertex operator:

$$
V_{\mathbf{q}}(a)=: e^{i \mathbf{q} \boldsymbol{\varphi}(a)}:
$$

which corresponds to the branch point with $\mathbf{q} \boldsymbol{\varphi}=\sum_{k} q_{k} \varphi_{k}$. From (2.12), (2.15) it is easy to determine the conformal dimension $\Delta$ of the operator $V_{\mathbf{q}}(a)$ :

$$
\begin{gathered}
\Delta=\sum \Delta_{k}=\sum\left(\frac{1}{2} q_{k}^{2}+\left(j-\frac{1}{2}\right) q_{k}\right)=\frac{n C_{3}}{24}\left(1-\frac{1}{n^{2}}\right), \\
C_{j}=-2\left(6 j^{2}-6 j+1\right),
\end{gathered}
$$

where $C=n C_{j}$ is the central charge of the Virasoro algebra of Laurent components of the total stress-energy tensor $T=\sum T_{k}$ of the system of fields $f_{k}, \phi_{k}$, $k=0, \ldots, n-1$.

Note that the relation

$$
\Delta=\frac{C}{24}\left(1-\frac{1}{n^{2}}\right)
$$

is the consequence of the general transformation law of the stress-energy tensor $T$ under the analytic change of coordinates in any conformal field theory with the central charge $C[6]$ :

$$
T(y)=\left(\frac{d Z}{d y}\right)^{2} T(Z)+\frac{C}{12}\left[\frac{d^{3} Z / d y^{3}}{d Z / d y}-\frac{3}{2}\left(\frac{d^{2} Z / d y^{2}}{d Z / d y}\right)^{2}\right]
$$

In coordinates $y$ defined in (2.2) $T(y)$ is regular at $y=0$ and then from (2.19) it follows that in $Z$-coordinates $T$ acquires a singularity at $Z=a$,

$$
T(Z)=C / 24(Z-a)^{-2}\left(1-1 / n^{2}\right),
$$

from which (2.18) follows.

\footnotetext{
${ }^{2}$ Recall that (2.13) and the analogous relations below have to be understood as the identities between various correlation functions
} 
Let us proceed now to the proof of the formulae (2.12), (2.13). We consider the operator product expansion (OPE) $f^{(\ell)}\left(Z^{\prime}\right) \phi^{(m)}(Z)$ in the vicinity of the branch point $^{3}$ :

$$
\begin{aligned}
f^{(\ell)}\left(Z^{\prime}\right) \phi^{(m)}(Z) & =\left(\frac{d y^{\prime}}{d Z^{\prime}}\right)^{j} f\left(y^{\prime}\right)\left(\frac{d y}{d Z}\right)^{1-j} \phi(y) \\
& =n^{-1}\left(y^{\prime}\right)^{j(1-n)}(y)^{(1-j)(1-n)}\left(y^{\prime}-y\right)^{-1} \\
& =n^{-1}\left(Z^{\prime}-Z\right)^{-1} \sum_{\ell=0}^{n-1}\left(y^{\prime} / y\right)^{\ell+j(1-n)} .
\end{aligned}
$$

Here with the help of (2.6) we have made the conformal transformation to the coordinates $y, \bar{y}$ and used the triviality of the OPE in these coordinates

$$
f\left(y^{\prime}\right) \phi(y)=\left(y^{\prime}-y\right)^{-1}+\text { Reg. terms . }
$$

From (2.10) and (2.20) follows that

$$
\begin{aligned}
f_{k}\left(Z^{\prime}\right) \phi_{m}(Z) & \simeq \delta_{k, m}\left(Z^{\prime}-Z\right)^{-1}\left(y^{\prime} / y\right)^{k+j(1-n)} \\
\simeq & \delta_{k, m}\left(\left(Z^{\prime}-Z\right)^{-1}+\frac{k+j(1-n)}{n}(Z-a)^{-1}\right)+O\left(\left(Z^{\prime}-Z\right)\right),
\end{aligned}
$$

and comparing with

$$
f_{k}\left(Z^{\prime}\right) \phi_{m}(Z)=\delta_{k, m}\left(Z^{\prime}-Z\right)^{-1}+: f_{k} \phi_{m}(Z):+O\left(\left(Z^{\prime}-Z\right)\right)
$$

for $k=m$ we get (2.12), (2.13).

Note that according to $(2.16)$ the product $f_{k}\left(Z^{\prime}\right) \phi_{k}(Z)$ near the branch point contributes to correlation functions the multiplier

$$
f_{k}\left(Z^{\prime}\right) \phi_{k}(Z) V_{\mathbf{q}}(a)=\left(Z^{\prime}-Z\right)^{-1}\left[\frac{Z^{\prime}-a}{Z-a}\right]^{q_{k}}: e^{i \varphi_{k}\left(z^{\prime}\right)} e^{-i \varphi_{k}(z)} e^{i \mathbf{q} \varphi(a)}:
$$

also confirming (2.21).

The formulae (2.12), (2.16) constitute the main results of this section.

\section{Interaction of Branch Points. $Z_{N}$-Symmetric Surfaces}

Let us apply now the results of Sect. 2 to the simple, but interesting enough case of the surfaces (1.3):

$$
y^{n}=\left(z-a_{1}\right) \ldots\left(z-a_{M}\right), \quad M=m n .
$$

For these surfaces the basis (2.9) diagonalizes all the operators $\hat{\pi}_{a_{i}}$ simultaneously and the formulae (2.15), (2.16) are correct globally, i.e. everywhere on the surface $X$. The number $M$ of the branch points in (1.3) is chosen to be an integer multiple of $n$ so that the infinity point would not be a branch point.

The basic quantity, playing the role of the partition function for analytic fields is the correlation function (dropping indices numerating the sheets):

$$
\begin{aligned}
& \left\langle f\left(Z_{1}\right) \ldots f\left(Z_{N_{J}}\right) \phi\left(Z^{\prime}\right) \ldots \phi\left(Z_{N_{1-j}}^{\prime}\right)\right\rangle \\
& \quad=\int D f D \phi f\left(Z_{1}\right) \ldots \phi\left(Z_{N_{1_{-}}}^{\prime}\right) \exp \left(\int_{X} f \bar{\partial} \phi d^{2} Z\right),
\end{aligned}
$$

\footnotetext{
${ }^{3}$ It is assumed in (2.20) that the value $y^{\prime}\left(z^{\prime}\right)(y(z))$ is taken on the sheet $\ell(m)$
} 
where the operators $f\left(Z_{i}\right)$ and $\phi\left(Z_{i}^{\prime}\right)$ stand to absorb all the zero modes of the operator $\bar{\partial}$. Thus $N_{i}$ is the number of holomorphic $j$-differentials on $X$. Choosing the basis $\left\{f_{\alpha}, \alpha=1, \ldots, N_{j}\right\}$ and $\left\{\phi_{\beta}, \beta=1, \ldots, N_{1-j}\right\}$ of holomorphic $j$ - and $(1-j)$ differentials, one can define the determinant of the Cauchy-Riemann operator, acting on $j$-differentials:

$$
\operatorname{det} \bar{\partial}_{j}=\frac{\left\langle f\left(Z_{1}\right) \ldots \phi\left(Z_{N_{1-j}}^{\prime}\right)\right\rangle}{\operatorname{det}\left\|f_{\alpha}\left(Z_{\gamma}\right)\right\| \operatorname{det}\left\|\phi_{\beta}\left(Z_{\delta}^{\prime}\right)\right\|} .
$$

Here $N_{j} \times N_{j}$ matrix $\left\|f_{\alpha}\left(Z_{\gamma}\right)\right\|$ has the element $f_{\alpha}\left(Z_{\gamma}\right)$ at the intersection of the column $\gamma$ and the row $\alpha$ and analogously for $\left\|\phi_{\beta}\left(Z_{\delta}^{\prime}\right)\right\|$. According to the basic theorem of papers $[7,8]^{4}$, the quantity (3.2) can be used to compute the determinant of the Laplace operator $\Delta_{j}$, acting on $j$-differentials:

$$
\operatorname{det}^{\prime} \Delta_{j}=\left|\operatorname{det} \bar{\partial}_{j}\right|^{2} \operatorname{det} M_{j} \operatorname{det} M_{1-j} \exp \left(C_{j} S_{L}\right),
$$

where $M_{j}^{\alpha \beta}=\int f_{\alpha} \bar{f}_{\beta} d Z \wedge d \bar{Z}$ is the matrix of scalar products of holomorphic $j$ differentials in the metric (1.2) and $S_{L}$ is the Liouville action for this metric. Since the latter does not depend on $a_{i}$ one can represent (3.3) in the form

$$
\operatorname{det}^{\prime} \Delta_{j}=\int d^{2} Z_{1} \ldots d^{2} Z_{N_{1-3}}^{\prime}\left|\left\langle f\left(Z_{1}\right) \ldots \phi\left(Z_{N_{1-}}^{\prime}\right)\right\rangle\right|^{2},
$$

where a constant (possibly infinite) multiplier has been dropped. To derive (3.4) we have used the fact that (3.2) does not depend on $Z_{\gamma}$ and $Z_{\delta}^{\prime}$. We also recall that

$$
\operatorname{det}^{\prime} \Delta_{j}=\operatorname{det}^{\prime} \Delta_{1-j} \text {. }
$$

Let us proceed now to the calculation of the correlation functions (3.1) by using the rules (2.12), (2.16). In accordance with (2.15) each field $\varphi_{m}$ has the charge $2 j-1$ at infinity. Accordingly only those correlators will be non-zero, for which the total charge of all operators with respect to each field $\varphi_{m}$ is equal to $1-2 j$. Since the operators $f_{k}, \phi_{k}$ and the branch point operators have charges $\pm \delta_{k, m}$ and $q_{m}$ respectively, we get the condition

$$
d_{j, k}=N\left(f_{k}\right)-N\left(\phi_{k}\right)=1-2 j-m n q_{k},
$$

$k=0,1, \ldots, n-1$. Here $N\left(f_{k}\right)$ and $N\left(\phi_{k}\right)$ denote the numbers of operators $f_{k}$ and $\phi_{k}$ in the correlation function under consideration. Note that by summing (3.6) over $k$ we obtain the Riemann-Roch theorem:

$$
\operatorname{ind}\left(\bar{\partial}_{j}\right)=N_{j}-N_{1-j}=(2 j-1)(p-1)
$$

where $p=1-n+m n(n-1) / 2$ is the genus of the surface (1.3). The latter follows from the Riemann-Hurwitz formula stating that the genus of a surface $X$ which is an $n$-sheeted branch covering of $\mathbb{C P}^{1}$ with $L$ branch points $a_{i}$ of orders $n_{i}$ is given by

$$
P=1-n+\sum_{i=1}^{L} \frac{n_{i}-1}{2} .
$$

Thus the formulae (2.12), (2.16) and the rules (3.6) provide the full description of all correlation functions of analytic fields on surfaces (1.3). For the determinants of the

${ }^{4}$ As was pointed out in [17] this theorem is a particular case of the general formula for the determinants of Dirac operators on arbitrary compact manifolds, due to Bismut and Freed [18] 
Laplace operators $\Delta_{j}$ in the metric (1.2) we get the integral representation (3.4) which is analogous to the feigin-Fuchs representation, used by Dotsenko and Fateev [3] to construct correlation functions of primary fields for the minimal conformal models of [6].

For the important particular case $j=1, d_{1, k}=m(n-k-1)-1$, making use of (3.5) we get

$$
\begin{aligned}
\operatorname{det}^{\prime} \Delta_{0}= & \int \prod_{k=0}^{n-2} \prod_{i=1}^{d_{1, k}} d^{2} Z_{i, k}\left|\left\langle\prod_{i, k} f_{k}\left(Z_{i, k}\right) \phi_{n-1}(Z)\right\rangle\right|^{2} \\
= & \prod_{k=0}^{n-2} \int \prod_{i=1}^{d_{1, k}} d^{2} Z_{i, k} \mid \prod_{i<j}^{d_{1, k}}\left(Z_{i, k}-Z_{j, k}\right) \\
& \times\left.\prod_{i=1}^{d_{1, k}}\left[y\left(Z_{i, k}\right)\right]^{k+1-n} \prod_{\alpha<\beta}^{m \cdot n}\left(a_{\alpha}-a_{\beta}\right)\left(\frac{k+1-n}{n}\right)^{2}\right|^{2} .
\end{aligned}
$$

In the second equality we have dropped the infinite constant $\int d^{2} Z$, using the fact that $\phi_{(n-1)}(Z)$ absorbe the constant scalar zero mode and the correlation function in (3.9) does not depend on $Z$.

\section{Hyperelliptic Surfaces and $Z_{\mathbf{2}}$-Twists}

Let us now concentrate our attention on the case $n=2$ of hyperelliptic surfaces, defined by the equation

$$
y^{2}=\left(Z-a_{1}\right) \ldots\left(Z-a_{2 p+2}\right)
$$

in $\mathbb{C}^{2}=(y, Z)$. Calculation of the determinant of the Laplace operator on such a surface is of particular interest on connection with the problem of defining the correlation functions of spin operators in the Ashkin-Teller model or the orbifold $Z_{2}$-twist fields $[4,5]$.

The $Z_{2}$-twist operator $\sigma(a, \bar{a})$ in the theory of free massless real scalar field $x(Z, \bar{Z})$ with the action

$$
S(x)=1 / 2 \pi \int \partial x \bar{\partial} x d^{2} Z
$$

is characterized by the following boundary conditions on currents $J=\partial x, \bar{J}=\bar{\partial} x$ :

$$
\hat{\pi}_{a} J=-J, \quad \hat{\pi}_{a} \bar{J}=-\bar{J} .
$$

Herewith the number of possible boundary conditions $z$ on the field $x$ itself is infinite and to obtain the correlation function of $\sigma$ 's it is necessary to sum over all of them:

$$
G\left(\left\{a_{i}\right\}\right) \equiv\left\langle\sigma\left(a_{1}, \bar{a}_{1}\right) \ldots \sigma\left(a_{2 p+2}, \bar{a}_{2 p+2}\right)\right\rangle=\left.\sum_{3} \int D x\right|_{3} e^{-S(x)} .
$$

In each sector it is convenient to represent $x$ as a sum

$$
x=x_{\mathrm{c} \ell}+x_{\mathrm{qu}} .
$$

Here $x_{\mathrm{c} t}(Z, \bar{Z})$ is the solution of the classical equation of motion

$$
\partial \bar{\partial} x_{\mathrm{c} \ell}=0
$$

satisfying $z$, and $x_{\mathrm{qu}}$ is the quantum field subject to the same boundary conditions for all $\mathfrak{z}$

$$
\hat{\pi}_{a_{i} x_{\mathrm{qu}}}=-x_{\mathrm{qu}}, \quad i=1, \ldots, 2 p+2 .
$$


Then (4.3) reduces to the sum

$$
G\left(\left\{a_{i}\right\}\right)=\int D x_{\mathrm{qu}} e^{-S\left(x_{\mathrm{qu}}\right)}\left\{\sum_{z} e^{-S\left(x_{\mathrm{c}} t\right)}\right\}
$$

The first multiple here can be expressed through the determinant of the Laplace operator $\Delta_{0}$ on the surface (4.1) with the metric (1.2). Indeed, any function $\tilde{x}$ on this surface can be decomposed into the sum

$$
\tilde{x}=x_{+}+x_{-}, \quad \hat{\pi}_{a_{\imath}} x_{ \pm}= \pm x_{ \pm} .
$$

Using now the property

$$
S(\tilde{x})=S\left(x_{+}\right)+S\left(x_{-}\right)
$$

of the action $S$ in the metric (1.2) and identifying the field $x_{-}$with $x_{\text {qu }}$, we get

$$
\begin{aligned}
\left(\operatorname{det}^{\prime} \Delta_{0}\right)^{-1 / 2} & =\int D \tilde{x} e^{S(\tilde{x})}=\int D x_{+} e^{-S(x+)} \int D x_{-} e^{-S(x-)} \\
& =\int D x_{\mathrm{qu}} e^{-S\left(x_{\mathrm{qu}}\right)}
\end{aligned}
$$

because the integral over $x_{+}$obviously does not depend on $a_{i}$. As a result

$$
G\left(\left\{a_{i}\right\}\right)=\left(\operatorname{det}^{\prime} \Delta_{0}\right)^{-1 / 2}\left\{\sum_{3} e^{-S(x} e^{(3))}\right\} .
$$

It is useful to keep in mind that in the limit of the infinite radius of the orbifold only the term with $x_{c \ell}=0$ will survive in the sum of (4.10) and the correlation function will be fully determined by the determinant. We also remark that this multiplier contains all the perturbation theory $\sigma$-model contribution to the correlation function, because the sum in (4.10) describes the contribution of worldsheet instantons.

To calculate $\operatorname{det}^{\prime} \Delta_{0}$ we use (3.9) which for $n=2$ takes the form

$$
\begin{aligned}
\operatorname{det}^{\prime} \Delta_{0} & =\int \prod_{k=1}^{p} d^{2} Z_{k}\left|\left\langle f\left(Z_{1}\right) \ldots f\left(Z_{p}\right) \phi(Z)\right\rangle\right|^{2} \\
& =\int \prod_{k=1}^{p} d^{2} Z_{k}\left|\prod_{m<\ell}^{p}\left(Z_{m}-Z_{\ell}\right) \prod_{\ell=1}^{p} y^{-1}\left(Z_{\ell}\right) \prod_{i<j}\left(a_{i}-a_{j}\right)^{1 / 4}\right|^{2} .
\end{aligned}
$$

In order to transform this expression to the one obtained in [4] it is useful to return to (3.2), which for $j=1$ can be written as

$$
\left\langle f\left(Z_{1}\right) \ldots f\left(Z_{p}\right) \phi(Z)\right\rangle=\operatorname{det} \bar{\partial}_{1} \operatorname{det}\left\|\omega_{i}\left(Z_{j}\right)\right\| .
$$

Here we put the scalar zero mode equal to 1 and $p$ holomorphic 1-differentials $\omega_{i}$ are normalized as

$$
\oint_{a_{\imath}} \omega_{j} d Z=\delta_{i j},
$$

where $a_{i}, i=1 \ldots p$ is one half of $2 p$ independent homologically nontrivial oriented cycles $a_{i}, b_{i}$ on $X$ with the intersection matrix

$$
a_{i} \circ a_{j}=b_{i} \circ b_{j}=0, \quad i \neq j, \quad a_{i} \circ b_{j}=\delta_{i j} .
$$

It is well-known that the matrix $M_{1}^{i j}$ of scalar products of such differentials is equal to the imaginary part of the period matrix $\tau$ [19]:

$$
M_{1}^{i j}=\int \omega_{i} \bar{\omega}_{j} d^{2} Z=\operatorname{Im} \tau_{i j}
$$



Applying to the left and right parts of (4.12) the operator $\prod_{i=1}^{p} \oint_{a_{i}} d Z_{i}$, and using (4.11)
and (3.3), we get

$$
\begin{gathered}
\operatorname{det} \bar{\partial}_{1}=\operatorname{det} \bar{\partial}_{0}=\operatorname{det} K \prod_{i<j}\left(a_{i}-a_{j}\right)^{1 / 4}, \\
\operatorname{det}^{\prime} \Delta_{0}=|\operatorname{det} K|^{2} \prod_{i<j}\left|a_{i}-a_{j}\right|^{1 / 2} \operatorname{det} \operatorname{Im} \tau,
\end{gathered}
$$

where the element $K_{i j}$ of the $p \times p$ matrix $K$ is given by

$$
K_{i j}=\oint_{a_{i}} z^{j-1} y^{-1}(z) d z ; \quad i, j=1, \ldots, p .
$$

This is just the expression for $\operatorname{det}^{\prime} \Delta_{0}$ obtained in [4]. Note that the case $p=1$ was also treated in [5]. The methods used in [4] and [5] are analogous and totally differ from those of the present paper.

\section{Free Fermions on Hyperelliptic Surfaces and Theta Functions}

In this section we consider the analytic fields with half integer spin $j$ on hyperelliptic surfaces (4.1). For such $j$ 's the possibility arise to impose various boundary conditions on the fields $f$ and $\phi$ : they can be periodic or antiperiodic around basic cycles of the surface (4.1). Analyzing the order of the singularity of fields $f$ and $\phi$ at branching points for different boundary conditions, it is not difficult to show that their choice requires now two vertex operators, corresponding to the branch point:

$$
\begin{gathered}
V_{+}\left(a_{i}\right)=\exp i\left(-\frac{j}{2} \varphi_{0}+\frac{1-j}{2} \varphi_{1}\right), \\
V_{-}\left(a_{i}\right)=\exp i\left(\frac{1-j}{2} \varphi_{0}-\frac{j}{2} \varphi_{1}\right) .
\end{gathered}
$$

Recall that $f_{k}=e^{i \varphi_{k}}, \varphi_{k}=e^{-i \varphi_{k}}, k=0,1$. Herewith the total charge of all branch points with respect to each of the fields $\varphi_{0}, \varphi_{1}$ has to be an integer, and up to the change $\varphi_{0} \leftrightarrow \varphi_{1}$ there are $2^{2 p}$ possibilities, in accordance with the total number of different boundary conditions.

For example in the simplest case of a torus, $p=1$, there are four variants, the corresponding nonzero correlation functions being:

$$
\begin{gathered}
\left\langle V_{+}\left(a_{1}\right) V_{+}\left(a_{2}\right) V_{-}\left(a_{3}\right) V_{-}\left(a_{4}\right)\right\rangle \\
=\prod_{i<j}\left(a_{i j}\right)^{\frac{j(j-1)}{2}}\left(a_{12} \cdot a_{34}\right)^{1 / 4}=\operatorname{det}_{(1,0)} \bar{\partial}_{j}, \\
\left\langle V_{+}\left(a_{1}\right) V_{-}\left(a_{2}\right) V_{+}\left(a_{3}\right) V_{-}\left(a_{4}\right)\right\rangle \\
=\prod_{i<j}\left(a_{i j}\right)^{\frac{j(j-1)}{2}}\left(a_{13} a_{24}\right)^{1 / 4}=\operatorname{det}_{(0,0)} \bar{\partial}_{j}, \\
\left\langle V_{+}\left(a_{1}\right) V_{-}\left(a_{2}\right) V_{-}\left(a_{3}\right) V_{+}\left(a_{4}\right)\right\rangle \\
=\prod_{i<j}\left(a_{i j}\right)^{\frac{j(j-1)}{2}}\left(a_{14} a_{23}\right)^{1 / 4}=\operatorname{det}_{(0,1)} \bar{\partial}_{j}, \\
\left\langle f_{0}(z) \phi_{1}\left(z^{\prime}\right) \prod_{i=1}^{4} V_{+}\left(a_{i}\right)\right\rangle=y^{-j}(z) y^{j-1}\left(z^{\prime}\right) \prod_{i<j}\left(a_{i j}\right)^{\frac{j(j-1)}{2}+\frac{1}{4}}, a_{i j} \equiv a_{i}-a_{j} .
\end{gathered}
$$


The total charge of all operators $V_{ \pm}\left(a_{i}\right)$ in $(5.2 \mathrm{a}-\mathrm{c})$ is equal to $1-2 j$ with respect to each of the fields $\varphi_{0}, \varphi_{1}$, and there are no zero modes of $f$ and $\phi$ fields. On the other hand, under the boundary conditions corresponding to (5.2d) two zero modes appear and in order to absorb them the operators $f_{0}(z)$ and $\phi_{1}\left(z^{\prime}\right)$ were introduced. The index $\left(m^{\prime}, m^{\prime \prime}\right)$ of $\operatorname{det}_{\left(m^{\prime}, m^{\prime \prime}\right)} \bar{\partial}_{j}$ defines the boundary conditions: the fields $f$ and $\phi$ acquire the multiplier $(-1)^{m^{\prime}}\left((-1)^{m^{\prime \prime}}\right)$ when moved around the cycle $a(b)$ surrounding the points $a_{1}, a_{2}\left(a_{2}, a_{3}\right)$.

If we transform to the coordinates $u$, which are defined by $d u=y^{-1}(z) d z$, the torus surface above will turn into the parallelogram. The case $(5.2 \mathrm{~d})$ and the zero modes $f(z)=y^{-j}(z), \phi(z)=y^{j-1}(z)$ correspond to periodic boundary conditions and to constant zero modes in $u$-coordinates. Three other cases, described by $(5.2 \mathrm{a}-\mathrm{c})$ correspond to three types of antiperiodic boundary conditions.

Let us fix now $j=1 / 2$. Analysis of this case makes it possible to derive a number of identities on the theta functions of hyperelliptic surfaces. For this we shall use the general formula for the fermionic determinant $[8,11]$ :

$$
\operatorname{det}_{\mathbf{m}} \bar{\partial}_{1 / 2}\left(\operatorname{det} \bar{\partial}_{0}\right)^{1 / 2}=\theta_{\mathbf{m}},
$$

where the characteristic $\mathbf{m} \equiv\left(\mathbf{m}^{\prime}, \mathbf{m}^{\prime \prime}\right)$ defines the boundary conditions imposed on fermions, i.e. 1/2-differentials, $\theta_{\mathbf{m}}$ being the corresponding theta-constant. We recall that components of $p$-dimensional vectors $\mathbf{m}^{\prime}, \mathbf{m}^{\prime \prime}$ take values 0,1 in such a way that the fermion field acquires a multiplier $(-1)^{m^{\prime}}\left[\right.$ or $\left.(-1)^{m^{\prime \prime}}\right]$ when moved around the cycle $a_{i}$ (or $b_{i}$ ). The theta-function and the corresponding thetaconstant are defined by the period matrix (4.15) as follows [10]:

$$
\begin{aligned}
\theta_{\mathbf{m}}(\boldsymbol{\xi}) & =\sum_{\mathbf{n} \in \mathbb{Z}^{p}} \exp \left\{\pi i\left(\mathbf{n}+\frac{\mathbf{m}^{\prime}}{2}\right) \tau\left(\mathbf{n}+\frac{\mathbf{m}^{\prime}}{2}\right)^{t}\right. \\
& \left.+2 \pi i\left(\mathbf{n}+\frac{\mathbf{m}^{\prime}}{2}\right)\left(\boldsymbol{\xi}+\frac{\mathbf{m}^{\prime \prime}}{2}\right)^{t}\right\}, \\
\theta_{\mathbf{m}} & \equiv \theta_{\mathbf{m}}(0), \quad \theta_{\mathbf{m}, i} \equiv \partial \theta_{\mathbf{m}}(\boldsymbol{\xi}) /\left.\partial \xi^{i}\right|_{\xi=0}, \ldots
\end{aligned}
$$

In (5.3) it is assumed that there are no fermionic zero modes on a surface. If, on the contrary, zero modes are present the $\operatorname{det}_{\mathbf{m}} \partial_{1 / 2}$ has to be defined with the help of (3.1), (3.2). The relation (5.3) for such a case takes the form [2]:

$$
\left\langle\psi \bar{\psi}\left(z_{1}\right) \ldots \psi \bar{\psi}\left(z_{n}\right)\right\rangle\left(\operatorname{det} \bar{\partial}_{0}\right)^{1 / 2}=\theta_{\mathbf{m}, i_{1} \ldots i_{n}} \omega_{i_{1}}\left(z_{1}\right) \ldots \omega_{i_{n}}\left(z_{n}\right),
$$

where we have redenoted $f$ and $\phi$ by $\psi$ and $\bar{\psi}$. It is obvious that the number $n$ of $\psi$ zero modes coincide with that for $\bar{\psi}$. Computing in (5.3) and (5.5) the left-hand sides by the methods described above, one can obtain a lot of useful expressions for theta-constants and their derivatives.

We begin with (5.3). Since for $j=1 / 2$ the fields $\varphi_{0}$ and $\varphi_{1}$ have no charge at infinity, the correlation functions $\left\langle\prod_{i^{\prime}} V_{+}\left(a_{i^{\prime}}\right) \prod_{i^{\prime \prime}} V_{-}\left(a_{i n}\right)\right\rangle$ will not vanish if the number of $V_{+}$'s is equal to the number of $V_{-}$'s. Denoting the corresponding characteristic by the symbol $\left\{i^{\prime} \mid i^{\prime \prime}\right\}$ we get with the help of (5.1),

$$
\operatorname{det}_{\left\{i^{\prime} \mid i^{\prime \prime}\right\}} \bar{\partial}_{1 / 2}=\prod_{i^{\prime}<j^{\prime}}\left(a_{i^{\prime}}-a_{j^{\prime}}\right)^{1 / 8} \prod_{i^{\prime \prime}<j^{\prime \prime}}\left(a_{i^{\prime \prime}}-a_{j^{\prime \prime}}\right)^{1 / 8} \prod_{i^{\prime}, i^{\prime \prime}}\left(a_{i^{\prime}}-a_{i^{\prime \prime}}\right)^{-1 / 8} .
$$


Substitution of this expression into (5.3) using (4.16a) gives us the Thomae formula [10]:

$$
\theta_{\left\{i^{\prime} \mid i^{\prime \prime}\right\}}=\prod_{i^{\prime}<j^{\prime}}\left(a_{i^{\prime}}-a_{j^{\prime}}\right)^{1 / 4} \prod_{i^{\prime \prime}-j^{\prime \prime}}\left(a_{i^{\prime \prime}}-a_{j^{\prime \prime}}\right)^{1 / 4} \operatorname{det}^{1 / 2} K .
$$

We stress that the characteristics $\left\{i^{\prime} \mid i^{\prime \prime}\right\}$ exhaust all types of boundary conditions for which there are no fermion zero modes.

The cases corresponding to (5.5) are those with different numbers of $V_{+}$and $V_{-}$. The condition of integer-valuedness of the total charge of $V^{\prime} \mathrm{s}$ implies that the difference between numbers of $V_{+}$'s and $V_{-}$'s should be divisible by 4 ; for example for genus 2 we have 6 such characteristics $\mathbf{m}_{i}, i=1, \ldots, 6$. For each $\mathbf{m}_{i}$ there is one zero mode for $\psi$ and one for $\bar{\psi}$, the corresponding correlation functions being given by

$$
\begin{aligned}
\left\langle\bar{\psi}_{0}(z) \psi_{1}\left(z^{\prime}\right) V_{+}\left(a_{i}\right) \prod_{k \neq i} V_{-}\left(a_{k}\right)\right\rangle & \\
= & {\left[\left(z-a_{i}\right) y^{-1}(z)\left(z^{\prime}-a_{i}\right) y^{-1}\left(z^{\prime}\right)\right]^{1 / 2} } \\
\quad & \times \prod_{k}^{\prime}\left(a_{i}-a_{k}\right)^{-1 / 8} \prod_{k<\ell}^{\prime}\left(a_{k}-a_{\ell}\right)^{1 / 8} ; \quad k, \ell \neq i .
\end{aligned}
$$

The analog of (5.7) has the form

$$
\left(z-a_{i}\right) y^{-1}(z) \prod_{k<\ell}^{\prime}\left(a_{k}-a_{\ell}\right)^{1 / 4} \operatorname{det}^{1 / 2} K=\theta_{\mathbf{m}_{i}, j} \omega_{j}(z) ; \quad k, \ell \neq i .
$$

With the use of these identities we shall show in the next section that the BeilinsonManin formula for the measure in the bosonic string theory [12] at least for genus $p=2$ does not depend on the choice of the basic odd characteristic and does reduce to the expressions obtained in $[13,14]$.

\section{The Two-Loop Measure in the Bosonic String Theory}

In this section we calculate the two-loop measure for the model of closed oriented bosonic strings at the critical dimension $\mathscr{D}=26$ (ESVM) by using the methods of the preceding sections. We also show that the result we obtain can be transformed to the expressions found earlier by using theta-functions (12-14). We start with the general expression for the $p$-loop vacuum measure in ESVM, found in $[7,8]$ for $p>1$ :

$$
Z_{p}=\int_{\mathscr{M}_{p}} \prod_{i=1}^{3 p-3} d y_{i} \wedge d \bar{y}_{i}|F(y)|^{2} \operatorname{det}^{-13} \operatorname{Im} \tau, \quad F(y)=\operatorname{det} \bar{\partial}_{1}\left(\operatorname{det} \bar{\partial}_{0}\right)^{-13} .
$$

Here $y_{i}, \bar{y}_{i}$ are some complex analytic coordinates on the moduli space $\mathscr{M}_{p}$ of genus $p$ Riemann surfaces, which are determined by some basis $f_{i}(Z), i=1, \ldots, 3 p-3$ of holomorphic quadratic differentials on a Riemann surface, $\tau$ being its period matrix. This basis has to be used in the definition (3.2) for $\operatorname{det} \bar{\partial}_{-1}$ :

$$
\operatorname{det} \bar{\partial}_{-1}=\left\langle f\left(z_{1}\right) \ldots f\left(z_{3 p-3}\right)\right\rangle \operatorname{det}^{-1}\left\|f_{i}\left(z_{j}\right)\right\| \text {. }
$$

$\operatorname{det} \bar{\partial}_{0}$ in (6.1) is defined analogously with the help of the normalized basis (4.13) of holomorphic 1-differentials.

When $p=2$ all surfaces are hyperelliptic and can be defined by the equation

$$
y^{2}=\left(z-a_{1}\right) \ldots\left(z-a_{6}\right)
$$


in $\mathbb{C}^{2}=(y, z)$. The moduli space $\mathscr{M}_{2}$ can be parametrized by coordinates of any three branch points, say $a_{1}, a_{2}, a_{3}$ with the position of three others being fixed. Herewith $\mathscr{M}_{2}$ will be covered by the hyperplane $\mathbb{C}^{3}=\left(a_{1}, a_{2}, a_{3}\right) 6 !=720$ times and in order to find $Z_{2}$ one can integrate over full $\mathbb{C}^{3}$, dividing then the result by 720 . For the metric (1.2) we have already computed all the correlation functions entering the definitions of the determinants in (6.1) and it remains to find the appropriate basis $f_{i}(Z), i=1,2,3$ in (6.2). It is not difficult to check that this basis has the following form:

$$
f_{i}(z)=\left(z-a_{i}\right)^{-1} \prod_{k=4}^{6} \frac{a_{i}-a_{k}}{z-a_{k}}, \quad i=1,2,3 .
$$

The determinant in the denominator of (6.2) is given by:

$$
\begin{aligned}
& \prod_{i=1}^{3} d a_{i} \operatorname{det}^{-1}\left\|f_{i}\left(z_{j}\right)\right\| \\
& \quad=\frac{d a}{d v_{\mathrm{pr}}}\left(a_{45} a_{56} a_{64}\right)^{-1} \operatorname{det}^{-1}\left\|\left(z_{j}-a_{i}\right)^{-1}\right\| \prod_{j=1}^{3} \prod_{k=4}^{6} \frac{z_{j}-a_{k}}{a_{j}-a_{k}} \\
& \quad=\frac{d a}{d v_{\mathrm{pr}}} \prod_{i<j}^{3}\left(z_{i j}\right)^{-1} \prod_{k<\ell}^{6}\left(a_{k \ell}\right)^{-1} \prod_{i=1}^{3} y^{2}\left(z_{i}\right),
\end{aligned}
$$

with the notations:

$$
\begin{gathered}
d a \equiv \prod_{i=1}^{6} d a_{i}, \quad d v_{\mathrm{pr}} \equiv d a_{4} d a_{5} d a_{6}\left(a_{45} a_{56} a_{64}\right)^{-1}, \\
z_{i j}=z_{i}-z_{j}, \quad a_{i j}=a_{i}-a_{j} .
\end{gathered}
$$

The correlation function in the numerator of (6.2) can be computed by using the general rules (2.12), (2.16). For $j=2, n=2$ with account of (3.6) we find

$$
\begin{aligned}
\left\langle\prod_{i=1}^{3} f\left(z_{i}\right)\right\rangle & =\left\langle\prod_{i=1}^{3} f_{0}\left(z_{i}\right) \prod_{k=1}^{6} V_{(-1,-1 / 2)}\left(a_{k}\right)\right\rangle \\
& =\prod_{i<j}^{3} z_{i j} \prod_{i=1}^{3} y^{-2}\left(z_{i}\right) \prod_{k<\ell}^{6}\left(a_{k}-a_{\ell}\right)^{5 / 4} .
\end{aligned}
$$

The other factors in (6.1) have already been computed in Sect. 4 - the expressions (4.11), (4.16). Collecting all them together, one gets

$$
\begin{aligned}
Z_{2} & =\int \frac{d^{2} a}{d V_{\mathrm{pr}}}\left|\prod_{k<\ell}^{6} a_{k \ell}^{-3} \operatorname{det}^{-13} K\right|^{2} \operatorname{det}^{-13} \operatorname{Im} \tau \\
& =\int \frac{d^{2} a}{d V_{\mathrm{pr}}}\left|\prod_{k<\ell}^{6} a_{k \ell}^{-3}\right|^{2}\left[\int d^{2} z_{1} d^{2} z_{2}\left|z_{12} y^{-1}\left(z_{1}\right) y^{-1}\left(z_{2}\right)\right|^{2}\right]^{-13}
\end{aligned}
$$

where $d^{2} a \equiv d a \wedge d \bar{a}$ and $d V_{\mathrm{pr}} \equiv d v_{\mathrm{pr}} \wedge d \bar{v}_{\mathrm{pr}}$ denotes the volume element of the group of projective transformations.

Since permutations of points $a_{i}$ do not change the complex structure of the Riemann surface, the single valued coordinate on $\mathscr{M}_{2}$ transversal to the subvariety $a_{i}=a_{j}$ of surfaces with a degenerate handle is $y_{i j}=\left(a_{i}-a_{j}\right)^{2}$. In coordinates $a_{i}, a_{k}, y_{i j}$ $(k \neq j)$ the holomorphic part of the measure in (6.7) obviously has the second order 
pole at $y_{i j}=0$, in accordance with the general theorem $[7,8]$. The additional factor $\left(\log \left|y_{i j}\right|\right)^{-13}$ is supplied by the integral in the square brackets in (6.7). It is also not difficult to check that the measure (6.7) has the correct behaviour when a surface degenerates into two punctured tori.

Let us establish now the connection of (6.7) with the formulae obtained in [13, 14]. In these papers the space $\mathscr{M}_{2}$ was parametrized by the elements $\tau_{i k}, i \leqq k$ of the period matrix (4.15). We have to make a change of variables in (6.7). In particular, we have to replace (6.4) by the basis, related with variations of $\tau_{i k}$. It was shown in [13] that such a basis is formed by products of normalized 1-differentials (4.13), so that $\omega_{i} \omega_{k}$ corresponds to the variation of $\tau_{i k}$ :

$$
d \tau_{i k} \mapsto f=\omega_{i} \omega_{k} .
$$

Denoting the elements of the new basis by $\tilde{f}_{i}: \tilde{f}_{1}=\omega_{1}^{2}, \widetilde{f}_{2}=\omega_{2}^{2}, \tilde{f}_{3}=\omega_{1} \omega_{2}$, we find

$$
\begin{aligned}
& \frac{d a}{d v_{\mathrm{pr}}} \operatorname{det}^{-1}\left\|f_{i}\left(z_{j}\right)\right\|=d \tau \operatorname{det}^{-1}\left\|\tilde{f}_{i}\left(z_{j}\right)\right\| \\
&=d \tau\left(\operatorname{det}\left\|\omega_{\alpha}\left(z_{\beta}\right)\right\| \operatorname{det}\left\|\omega_{\alpha}\left(z_{\gamma}\right)\right\| \operatorname{det}\left\|\omega_{\alpha}\left(z_{\delta}\right)\right\|\right)^{-1} \\
&=d \tau \operatorname{det}^{3} K \prod_{i<j}^{3} z_{i j}^{-1} \prod_{i=1}^{3} y^{2}\left(z_{i}\right) ; \\
& \beta=1,2 ; \quad \gamma=2,3 ; \quad \delta=3,1 ; \quad d \tau \equiv d \tau_{11} d \tau_{22} d \tau_{12},
\end{aligned}
$$

where in the last equality we made a transformation from the basis $\omega_{i}$ to the basis formed by

$$
w_{i}=z^{i-1} y^{-1}(z), \quad i=1,2,
$$

with also the use of (4.17). Recalling (6.5), we obtain

$$
\frac{d a}{d v_{\mathrm{pr}}}=d \tau \operatorname{det}^{3} K \prod_{k<\ell}^{6}\left(a_{k}-a_{\ell}\right)
$$

and (6.7) takes the form

$$
Z_{2}=\int_{\mathscr{M}_{2}} d^{2} \tau\left|\prod_{k<\ell}^{6}\left(a_{k}-a_{\ell}\right)^{-2} \operatorname{det}^{-10} K\right|^{2}(\operatorname{det} \operatorname{Im} \tau)^{-13}
$$

Finally, by multiplying the identities (5.7) for all 10 even characteristics (having no zero modes) we find the relation

$$
\chi_{10}(\tau) \equiv \prod_{\text {even } \mathbf{m}} \theta_{\mathbf{m}}^{2}=\operatorname{det}^{10} K \prod_{k<\ell}^{6}\left(a_{k}-a_{\ell}\right)^{2},
$$

from which the resullt of $[13,14]$ follows:

$$
Z_{2}=\int_{\mathscr{M}_{2}} d^{2} \tau\left|\chi_{10}(\tau)\right|^{-2} \operatorname{det}^{-13} \operatorname{Im} \tau
$$

We proceed now to the verification of the formula obtained by Beilinson and Manin [12]. According to $[2,12]$ the measure in (6.1) can be computed under a given odd characteristic $*$ by using the relation:

$$
F=\frac{\operatorname{det}^{9}\left\|\boldsymbol{\omega}\left(R_{1}\right) \boldsymbol{\omega}^{\prime \prime}\left(R_{1}\right) \boldsymbol{\omega}\left(R_{2}\right) \ldots \omega\left(R_{p-1}\right)\right\|}{\left[\theta_{*, i} \omega_{i}^{\prime \prime}\left(R_{1}\right)\right]^{8} \operatorname{det}\left\|\mathbf{f}\left(R_{1}\right) \mathbf{f}^{\prime \prime}\left(R_{1}\right) \mathbf{f}^{(4)}\left(R_{1}\right) \mathbf{f}\left(R_{2}\right) \ldots \mathbf{f}^{\prime}\left(R_{p-1}\right) \mathbf{f}^{\prime \prime}\left(R_{p-1}\right)\right\|},
$$


where $\boldsymbol{\omega}$ is a column of $p$ normalized 1-differentials and $\mathbf{f}$ is a column of $3 p-3$ holomorphic 2-differentials, used to define complex coordinates $y_{i}$ on $\mathscr{M}_{p}$ in (6.1). The points $R_{1} \ldots R_{p-1}$ are $p-1$ zeroes of a holomorphic 1/2-differential with the characteristic $*$, and $F$ should not depend on the choice of $*$.

For $p=2$ there are 6 holomorphic 1/2-differentials

$$
\psi_{\alpha}(z)=\left[\left(z-a_{\alpha}\right) y^{-1}(z)\right]^{1 / 2}, \quad \alpha=1, \ldots, 6
$$

with different odd characteristics $\mathbf{m}_{\alpha}$. Each of them has a single $1^{\text {st }}$ order zero at the point $a_{\alpha}$. For $*=\mathbf{m}_{\alpha}$ the expression (6.15) takes the form

$$
F=\frac{\operatorname{det}^{9}\left\|\omega\left(a_{\alpha}\right) \omega^{\prime \prime}\left(a_{\alpha}\right)\right\|}{\left[\theta_{\mathbf{m}_{\alpha}, i} \omega_{i}^{\prime \prime}\left(a_{\alpha}\right)\right]^{8} \operatorname{det}\left\|\mathbf{f}\left(a_{\alpha}\right) \mathbf{f}^{\prime \prime}\left(a_{\alpha}\right) \mathbf{f}^{(4)}\left(a_{\alpha}\right)\right\|} .
$$

Let us show how it reduces to (6.12). We substitute into (6.17),

$$
\mathbf{f}=\left(\tilde{f}_{1}, \tilde{f}_{2}, \tilde{f}_{3}\right)^{t}, \quad \boldsymbol{\omega}=K^{-1} \mathbf{w},
$$

where $\tilde{f}_{i}$ and $w_{i}$ were defined in (6.8), (6.10), and we use the identity (5.9). Shifting in (6.17) $a_{\alpha}$ by a small $\delta$ and extracting the terms most singular in $\delta$, we find $\left(a_{\alpha}+\delta \equiv a\right)^{5}$ :

$$
\begin{gathered}
\operatorname{det}\left\|\boldsymbol{\omega}(a) \boldsymbol{\omega}^{\prime \prime}(a)\right\| \sim \delta^{-2} \operatorname{det}^{-1} K \prod_{\beta}^{\prime}\left(a_{\alpha}-a_{\beta}\right)^{-1}, \\
\operatorname{det}\left\|\mathbf{f}(a) \mathbf{f}^{\prime \prime}(a) \mathbf{f}^{(4)}(a)\right\| \underset{(6.9)}{\sim} \delta^{-6} \operatorname{det}^{-3} K \prod_{\beta}^{\prime}\left(a_{\alpha}-a_{\beta}\right)^{-3}, \\
\theta_{\mathbf{m}_{\alpha}, i} \omega_{i}^{\prime \prime}(a) \sim \delta^{-3 / 2} \operatorname{det}^{1 / 2} K \prod_{\beta}^{\prime}\left(a_{\alpha}-a_{\beta}\right)^{-1 / 2} \prod_{\beta<\beta^{\prime}}^{\prime}\left(a_{\beta}-a_{\beta^{\prime}}\right)^{1 / 4} ; \\
\beta, \beta^{\prime} \neq \alpha .
\end{gathered}
$$

It follows that the power of $\delta$ in the numerator of (6.17) is the same as that in the denominator, and for $\delta \rightarrow 0,(6.17)$ has a finite limit

$$
F=\operatorname{det}^{-10} K \prod_{k<\ell}^{6}\left(a_{k}-a_{\ell}\right)^{-2}
$$

which does not depend on the initial choice of $\mathbf{m}_{\alpha}($ !). After substitution into (6.1) it gives (6.12), which reduces then to (6.14).

We end this section by giving for the $p=1$ the representations for the measure analogous to (6.7) for the case of ESVM and for the model of closed oriented superstrings. Defining the general genus 1 surface by the equation

$$
y^{2}=\left(z-a_{1}\right) \ldots\left(z-a_{4}\right)
$$

in $\mathbb{C}^{2}=(y, z)$, we find for the ESVM:

$$
Z_{1}=\int \frac{d^{2} a}{d V_{\mathrm{pr}}}\left|\prod_{k<\ell}^{4} a_{k \ell}^{-3}\right|^{2}\left[\int d^{2} z|y(z)|^{-2}\right]^{-14} .
$$

To compute the superstring measure one has to sum over all boundary conditions. Periodic conditions obviously don't contribute to the vacuum amplitude, and the

\footnotetext{
${ }^{5}$ The appearance of various singularities when $\delta \rightarrow 0$ is related to the fact that the coordinates $\delta, \bar{\delta}$
} are singular in the vicinity of the branch point $\delta=0$ 
three types of antiperiodic conditions as was explained in Sect. 5 are in one to one correspondence with the three possibilities to form two pairs $\left(a_{s}, a_{t}\right)$ and $\left(a_{u}, a_{v}\right)$ out of 4 branch points $a_{k}, k=1,2,3,4$.

The contribution to the measure from the sector with the conditions $\{s, t \mid u, v\}$ imposed on the left fermions and $\{r, p \mid q, w\}$ imposed on the right fermions can easily be computed with the use of (5.2) and is found to be

$$
\begin{aligned}
Z_{1}^{S S, \text { b.c. }}= & \int \frac{d^{2} a}{d V_{\mathrm{pr}}}\left|\prod_{k<\ell}^{4} a_{k \ell}^{-2}\right|^{2}\left[\int d^{2} z|y(z)|^{-2}\right]^{-6} \\
& \times\left(a_{s}-a_{t}\right)\left(a_{u}-a_{v}\right)\left(\bar{a}_{r}-\bar{a}_{p}\right)\left(\bar{a}_{q}-\bar{a}_{w}\right) .
\end{aligned}
$$

The relative weight of each contribution is determined unambiguously by the condition of the cancellation of tachyons, which are second order poles over $a_{k \ell}$ in $(6.22)^{6}$, and we obtain the result of Green and Schwarz [16]:

$$
\begin{aligned}
Z_{1}^{S S}= & \int \frac{d^{2} a}{d V_{\mathrm{pr}}}\left|\prod_{k<\ell}^{4} a_{k \ell}^{-2}\right|^{2}\left[\int d^{2} z|y(z)|^{-2}\right]^{-6} \\
& \times\left|a_{12} \cdot a_{34}-a_{13} a_{24}-a_{14} a_{32}\right|^{2}=0,
\end{aligned}
$$

as a consequence of the Riemann identity which takes the form

$$
a_{12} a_{34}-a_{13} a_{24}-a_{14} a_{32} \equiv 0 \text {. }
$$

Finally, I hope that the representation of the type of $(6.7)$ can be generalized to the theory of open strings.

\section{Twist Fields and $\bar{\partial}-$ Operator}

In this section we shall generalize the results of [5] and apply our methods to the calculation of multitwist correlation functions describing the string propagation in the orbifold background. Analogously to the $Z_{2}$-case, the operators of $Z_{N}$-twists $\sigma_{K}(a, \bar{a}), K=1, \ldots, N-1$, defined in the theory of a complex scalar field $X(Z, \bar{Z})$ with the action

$$
S(X)=\int\left(\partial X \bar{\partial} X^{*}+\bar{\partial} X \partial X^{*}\right) d^{2} z
$$

are characterized by the boundary conditions

$$
\begin{array}{cc}
\hat{\pi}_{a} J=\exp \frac{2 \pi i K}{N} J, & \hat{\pi}_{a} \bar{J}=\exp \frac{2 \pi i K}{N} \bar{J}, \\
\hat{\pi}_{a} J^{*}=\exp \frac{-2 \pi i K}{N} J^{*}, & \hat{\pi}_{a} \bar{J}^{*}=\exp \frac{-2 \pi i K}{N} \bar{J}^{*},
\end{array}
$$

which are imposed on the currents

$$
J=\partial X, \quad J^{*}=\partial X^{*}, \quad \bar{J}=\bar{\partial} X, \quad \bar{J}^{*}=\bar{\partial} X^{*} .
$$

We recall that $\hat{\pi}_{a}$ in (7.2) denotes the operation of the analytic continuation of correlation functions containing operators (7.3) along the contour surrounding the twist operator $\sigma(a, \bar{a})$.

${ }^{6}$ This is the well-known GOS projection [15] 
As in the case of $Z_{2}$-twists, any correlation function of the operators $\sigma_{K}$ is the product of two terms [5]:

$$
\left\langle\prod_{i=1}^{M} \sigma_{K_{i}}\left(a_{i}, \bar{a}_{i}\right)\right\rangle=Z_{\mathrm{qu}}\left(\left\{a_{i}\right\}\right) Z_{\mathrm{inst}}\left(\left\{a_{i}\right\}\right),
$$

where the first is given by the integral

$$
Z_{\mathrm{qu}}\left(\left\{a_{i}\right\}\right)=\int \mathscr{D} X_{\mathrm{qu}} \mathscr{D} X_{\mathrm{qu}}^{*} \exp \left[-S\left(X_{\mathrm{qu}}\right)\right]
$$

over the field $X_{\mathrm{qu}}$, satisfying for all $j$ the conditions,

$$
\begin{gathered}
\hat{\pi}_{a_{j}} X_{\mathrm{qu}}=\exp \frac{2 \pi i K_{j}}{N} X_{\mathrm{qu}}, \\
\hat{\pi}_{a_{j}} X_{\mathrm{qu}}^{*}=\exp \frac{-2 \pi i K_{j}}{N} X_{\mathrm{qu}}^{*} .
\end{gathered}
$$

The second multiplier in (7.4) describes the contribution of classical string trajectories, connecting the points of the orbifold, which corresponds to the operators $\sigma_{\mathbf{K}_{i}}$ :

$$
Z_{\mathrm{inst}}=\sum_{\left\{X_{\mathrm{c} 1}\right\}} \exp \left[-S\left(X_{\mathrm{cl}}\right)\right], \quad \partial \bar{\partial} X_{\mathrm{cl}}=0
$$

This quantity, in contrast to $Z_{\text {qu }}$, depends essentially on the global structure of the orbifold [5], and in the limit of infinite radius of the orbifold only the zero-action trajectories will contribute. We remark also that singlevaluedness of the field $X(Z, \bar{Z})$ at infinity involves the condition

$$
\sum_{i=1}^{M} K_{i}=0(\bmod N)
$$

which will be supposed to be satisfied in the following.

The correlation functions (7.4) for $M=4$ and $K_{1}=K_{3}=N-K_{2}=N-K_{4}$ were obtained in [5] for various orbifolds. The initial step in the calculation of $Z_{\mathrm{qu}}\left(a_{1}, \ldots, a_{4}\right)$ was the determination of the Green functions $\left\langle J(z) \overline{J^{*}\left(z^{\prime}\right)}\right\rangle$, $\left\langle J(z) J^{*}\left(z^{\prime}\right)\right\rangle$, in the presence of the operators $\sigma_{K_{i}}\left(a_{i}, \bar{a}_{i}\right)$, from the conditions of singlevaluedness, holomorphity and known orders and positions of all the singularities. The stress-energy tensor $\left\langle T_{\mathrm{qu}}(z)\right\rangle$ was then extracted from the operator product expansion of the currents. In accordance with [6] the residue of $\left\langle T_{\mathrm{qu}}(z)\right\rangle$ at $Z=a_{i}$ is equal to $\partial \ln Z_{\mathrm{qu}} / \partial a_{i}$. Integrating over $a_{i}$ the authors of [5] found $Z_{\mathrm{qu}}$ itself. This calculation, being very clear in its idea, is technically rather involved, especially in the case $K_{1} \neq K_{2}$ or when the number $M$ of $\sigma_{K}$-operators is greater than four.

Below we shall show that the transition to the analytic fields cause essential simplifications and allows us to construct the universal integral representation for all correlation functions of $\sigma_{K}$ 's for any $M$.

The transition to the $1^{\text {st }}$ order formalism is performed with the help of the analog of (3.3), which we give here without proof:

$$
Z_{\mathrm{qu}}^{-2}\left(\left\{a_{i}\right\}\right)=\left|\operatorname{det} \bar{\partial}_{*}\right|^{2} \operatorname{det} \hat{N}|\operatorname{det} \bar{\partial}|^{2} \operatorname{det} \widehat{N}_{*},
$$


where the operator $\bar{\partial}\left(\bar{\partial}_{*}\right)$ acts on scalar fields, subject to the conditions [7.6 $\left.a(b)\right]$; $\hat{N}\left(\hat{N}_{*}\right)$ is the matrix of scalar products of holomorphic 1-differentials, satisfying (7.6a(b)). Det $\bar{\partial}$ in (7.9) is defined analogously to (3.2) by using a pair of anticommuting analytic fields - the scalar $\phi$ and 1-differential $\omega^{*}$ satisfying respectively the conditions (7.6a) and (7.6b) - and having the action:

$$
S=\int \omega^{*} \bar{\partial} \phi d^{2} z .
$$

As in Sect. 3, in order to determine the partition function of the fields $\omega^{*}, \phi$, we have to consider the vacuum average in which operators $\omega^{*}$ and $\phi$ absorb all the zero modes of $\bar{\partial}$, and divide it by the appropriate zero modes determinant as in (3.2). Obviously there are no scalar zero modes satisfying (7.6a). The number of holomorphic 1-forms satisfying (7.6b) as will be shown below equals

$$
n_{*}=\left(\sum_{i=1}^{M} \frac{K_{i}}{N}\right)-1
$$

Thus by choosing a basis $\omega_{1}^{*}(z) \ldots \omega_{n_{*}^{*}}^{*}(z)$ of such forms, we can define

$$
\operatorname{det} \bar{\partial}=\left\langle\omega^{*}\left(z_{1}\right) \ldots \omega^{*}\left(z_{n_{*}}\right)\right\rangle \operatorname{det}^{-1}\left\|\omega_{i}^{*}\left(z_{j}\right)\right\| .
$$

Det $\bar{\partial}_{*}$ is defined analogously by using a pair of fields $\omega, \phi^{*}$ satisfying (7.6a) and (7.6b) respectively. The number $n$ of zero modes of $\omega$ for this case equals

$$
n=\left(\sum_{i=1}^{M} \frac{N-K_{i}}{N}\right)-1
$$

It is convenient to transform (7.8) to the form [cf. (3.2)-(3.4)]:

$$
\begin{aligned}
Z_{\mathrm{qu}}^{-2}\left(\left\{a_{i}\right\}\right) & =Z\left(\left\{a_{i}\right\}\right) Z_{*}\left(\left\{a_{i}\right\}\right), \\
Z\left(\left\{a_{i}\right\}\right) & =\left|\operatorname{det} \bar{\partial}_{*}\right|^{2} \operatorname{det} \hat{N} \\
& =\int d^{2} z_{1} \ldots d^{2} z_{n}\left|\left\langle\omega\left(z_{1}\right) \ldots \omega\left(z_{n}\right)\right\rangle\right|^{2}, \\
Z_{*}\left(\left\{a_{i}\right\}\right) & =|\operatorname{det} \bar{\partial}|^{2} \operatorname{det} \hat{N}_{*} \\
& =\int d^{2} z_{1} \ldots d^{2} z_{n_{*}}\left|\left\langle\omega^{*}\left(z_{1}\right) \ldots \omega^{*}\left(z_{n_{*}}\right)\right\rangle\right|^{2} .
\end{aligned}
$$

We proceed now to the calculation of the correlation functions entering $Z$ and $Z_{*}$, and begin with $Z_{*}$. The key point is that again as in Sect. 2 the primary conformal field $V_{i}\left(a_{i}\right)$ corresponds to each singular point $a_{i}$ of the fields $\omega^{*}, \phi$. It has the charge $-\frac{K_{i}}{N}$ with respect to the current $\left(\mathfrak{b}^{*}=: \omega^{*} \phi\right.$ :, i.e. the following OPE takes place:

$$
\mathfrak{b}^{*}(z) V_{i}\left(a_{i}\right)=-\frac{K_{i}}{N}\left(z-a_{i}\right)^{-1} V_{i}\left(a_{i}\right)+\text { Reg. terms . }
$$

The latter can easily be derived by comparing the expansion

$$
\omega^{*}\left(z^{\prime}\right) \phi(z) V_{i}\left(a_{i}\right)=\left[\left(z^{\prime}-z\right)^{-1}+\left(\mathfrak{b}^{*}(z)+O\left(z^{\prime}-z\right)\right] V_{i}\left(a_{i}\right)\right.
$$


with the relation

$$
\begin{aligned}
\omega^{*}\left(z^{\prime}\right) \phi(z) V_{i}\left(a_{i}\right) \\
=\left(z^{\prime}-z\right)^{-1}\left(z^{\prime}-a_{i}\right)^{-\frac{K_{i}}{N}}\left(z-a_{i}\right)^{\frac{K_{i}}{N}}: \omega^{*}\left(z^{\prime}\right) \phi(z) V_{i}\left(a_{i}\right): \\
=\left[\left(z^{\prime}-z\right)^{-1}-\frac{K_{i}}{N}\left(z-a_{i}\right)^{-1}\right] V_{i}\left(a_{i}\right) \\
\quad+:\left(\mathfrak{G}^{*}(z) V_{i}\left(a_{i}\right):+O\left(z^{\prime}-z\right),\right.
\end{aligned}
$$

which is a consequence of (7.6).

From (7.14) the following bosonization rules follow:

$$
\begin{gathered}
\omega^{*}=: e^{i \varphi}:, \quad \phi=: e^{-i \varphi}:, \quad \mathfrak{G}^{*}=i \partial \varphi, \quad V_{j}=: \exp \left(-i \frac{K_{j}}{N} \varphi\right):, \\
T=-\omega^{*} \partial \phi=\frac{1}{2}\left[:\left(\mathfrak{G}^{*}\right)^{2}:-\partial \mathfrak{6}\right],
\end{gathered}
$$

where the analytic scalar field $\varphi(z)$ is supposed to be normalized as

$$
\left\langle\varphi(z) \varphi\left(z^{\prime}\right)\right\rangle=-\ln \left(z-z^{\prime}\right)
$$

and has the charge 1 at infinity, i.e.

$$
\mathfrak{G}^{*}(z) \sim-z^{-1}, \quad z \rightarrow \infty .
$$

This implies that the total charge of all operators in any nonzero correlation function has to be equal to -1 , and therefore in the average

$$
\begin{aligned}
& \left\langle\omega^{*}\left(z_{1}\right) \ldots \omega^{*}\left(z_{n_{*}}\right) \prod_{i=1}^{M} V_{i}\left(a_{i}\right)\right\rangle \\
& =\prod_{k<\ell}^{n}\left(z_{k}-z_{\ell}\right) \prod_{k, i}\left(z_{k}-a_{i}\right)^{-K_{i} / N} \prod_{i<j}^{M}\left(a_{i}-a_{j}\right)^{K_{\imath} K_{J} / N^{2}},
\end{aligned}
$$

which we have computed using (7.15), (7.16), the number $n_{*}$ of $\omega^{*}$ operators has to be equal to (7.10).

The correlation function contained in $Z$ can be computed in the same way, which amounts to replacing $K_{i}$ by $N-K_{i}$ :

$$
\begin{aligned}
& \left\langle\omega\left(z_{1}\right) \ldots \omega\left(z_{n}\right) \prod_{i=1}^{M} V_{i}^{*}\left(a_{i}\right)\right\rangle \\
& \quad=\prod_{k<\ell}^{n}\left(z_{k}-z_{\ell}\right) \prod_{k, i}\left(z_{k}-a_{i}\right)^{-\frac{N-K_{i}}{N}} \prod_{i<j}^{M}\left(a_{i}-a_{j}\right)^{\left(N-K_{i}\right)\left(N-K_{J}\right) / N^{2}},
\end{aligned}
$$

where we have denoted by $V_{i}^{*}$ the operators corresponding to the singular points $a_{i}$ of the fields $\omega, \phi^{*}$. The number $n$ is determined by (7.12), in the same way as for $n_{*}$.

The dimensions of the operators $V_{i}$ and $V_{i}^{*}$ coincide and are given by

$$
\Delta_{K_{\imath}}=-\frac{1}{2} \frac{K_{i}}{N}\left(1-\frac{K_{i}}{N}\right),
$$

which is a consequence of the general relation $\Delta(q)=\frac{1}{2} q(q+1)$ for the dimension of the operator $V_{q}=$ :expiq $\varphi$ :. Recalling (7.8) one finds that the dimensions $h_{k}$ and $\bar{h}_{k}$ 
of the twist operator are equal to

$$
h_{K}=\bar{h}_{K}=\frac{K(N-K)}{2 N^{2}}
$$

in accordance with [5].

Thus we have constructed a simple integral representation described by (7.13), (7.18), and (7.19) for the quantum part of the twist-field correlation functions. Now we can proceed to the calculation of the structure constants of the twist-field operator algebra. First we note that for the correlation function

$$
\left\langle\prod_{i=1}^{M} \sigma_{K_{\imath}}\left(a_{i}, \bar{a}_{i}\right)\right\rangle_{\mathrm{qu}}=x^{M-2}\left[Z\left(\left\{a_{i}\right\}\right) Z_{*}\left(\left\{a_{i}\right\}\right)\right]^{-1 / 2}
$$

(where we have restored the normalization constant $x$ ) there are three possibilities to satisfy (7.8) when $M=4$ :

$$
\sum_{i=1}^{4} K_{i}=\ell \cdot N, \quad \ell=1,2,3 .
$$

Two of them $-\ell=1$ and $\ell=3$ - are related by the change

$$
K_{i} \rightarrow N-K_{i} \text {. }
$$

From the representation (7.9) it is obvious that all the correlation functions of $\sigma_{K}$ are invariant with respect to (7.23). So it is sufficient to consider the cases $\ell=1,2$. For the same reason the structure constants $C_{K_{1} K_{2} K_{3}}$ of the operator algebra of $\sigma_{K}$ 's, which determine the three-point couplings

$$
\left\langle\sigma_{K_{1}}(0) \sigma_{K_{2}}(1) \sigma_{K_{3}}(\infty)\right\rangle_{\mathrm{qu}}=\varkappa C_{K_{1} K_{2} K_{3}} \equiv C_{K_{1} K_{2} K_{3}}^{\mathrm{qu}}
$$

are also invariant with respect to (7.23):

$$
C_{K_{1} K_{2} K_{3}}^{\mathrm{qu}}=C_{N-K_{1}, N-K_{2}, N-K_{3}}^{\mathrm{qu}} .
$$

Hence it is sufficient to compute $C_{K_{1} K_{2} K_{3}}^{\mathrm{qu}}$ for $K_{1}+K_{2}+K_{3}=2 N$. It is possible to show that the integral representation $(7.13)-(7.19)$ gives the correct relative normalization of the three-point functions. For $K_{1}+K_{2}+K_{3}=2 N$ the numbers $n_{*}$ and $n$ in (7.13) for the factors $Z$ and $Z_{*}$ of (7.22) for the function

$$
\left\langle\sigma_{K_{1}}\left(a_{1}, \bar{a}_{1}\right) \sigma_{K_{2}}\left(a_{2}, \bar{a}_{2}\right) \sigma_{K_{3}}\left(a_{3}, \bar{a}_{3}\right)\right\rangle,
$$

are equal respectively to 1 and 0 . Therefore

$$
\begin{gathered}
Z_{*}\left(a_{1}, a_{2}, a_{3}\right)=\int d^{2} z\left|\left\langle\omega_{*}(z) V_{K_{1}}\left(a_{1}\right) V_{K_{2}}\left(a_{2}\right) V_{K_{3}}\left(a_{3}\right)\right\rangle\right|^{2}, \\
Z\left(a_{1}, a_{2}, a_{3}\right)=\left|\left\langle V_{N-K_{1}}^{*}\left(a_{1}\right) V_{N-K_{2}}^{*}\left(a_{2}\right) V_{N-K_{3}}^{*}\left(a_{3}\right)\right\rangle\right|^{2} .
\end{gathered}
$$

On substituting $a_{1}=0, a_{2}=1$ and $a_{3}=\infty$, we get

$$
\begin{aligned}
C_{K_{1} K_{2} K_{3}}^{-2} & =\int d^{2} z|z|^{-2 K_{1} / N}|z-1|^{-2 K_{2} / N} \\
& =\pi \prod_{i=1}^{3} \Gamma\left(1-K_{i} / N\right) / \Gamma\left(K_{i} / N\right) K_{1}+K_{2}+K_{3}=2 N .
\end{aligned}
$$


To find the absolute normalization $\varkappa$ of the structure constants, it is necessary to calculate also the four-point functions. We shall restrict ourselves to the case

$$
K_{1}+K_{2}+K_{3}+K_{4}=2 N
$$

and assume that

$$
K_{1}+K_{2}>N
$$

Herewith there is one integration in both $Z$ and $Z_{*}$. Putting $a_{1}, a_{2}, a_{3}$, and $a_{4}$ respectively at $0, x, 1$, and $\infty$, we obtain (see Appendix):

$$
\begin{aligned}
& Z_{*}\left(\left\{K_{i}\right\} \mid x\right)=\int d^{2} z|z|^{-2 K_{1} / N}|z-x|^{-2 K_{2} / N} \\
& \times|z-1|^{-2 K_{3} / N}|x|^{2 K_{1} K_{2} / N^{2}}|x-1|^{2 K_{2} K_{3} / N^{2}} \\
& =|x|^{2 K_{1} K_{2} / N^{2}}|1-x|^{2 K_{2} K_{3} / N^{2}}\left(C_{K_{1}, K_{2}, K_{3}+K_{4}}^{-2}|x|^{2\left(1-\frac{K_{1}+K_{2}}{N}\right)}\right. \\
& \times\left|F\left(1-\frac{K_{1}}{N}, \frac{K_{3}}{N}, \frac{K_{3}+K_{4}}{N}, x\right)\right|^{2} \\
& \left.+C_{K_{3}, K_{4}, K_{1}+K_{2}}^{-2}\left|F\left(1-\frac{K_{4}}{N}, \frac{K_{2}}{N}, \frac{K_{1}+K_{2}}{N}, x\right)\right|^{2}\right), \\
& Z\left(\left\{K_{i}\right\} \mid x\right)=Z_{*}\left(\left\{N-K_{i}\right\} \mid x\right) .
\end{aligned}
$$

Formulae (7.22), (7.28) give the quantum part of the four point correlation functions of the operators $\sigma_{K}$ for the case (7.27). It is not difficult to check that for $x \rightarrow 0$ the most singular part of the function (7.22) has the form

$$
\begin{aligned}
& \left\langle\sigma_{K_{1}}(0) \sigma_{K_{2}}(x) \sigma_{K_{3}}(1) \sigma_{K_{4}}(\infty)\right\rangle_{\mathrm{qu}}=x^{2}\left(Z \cdot Z_{*}\right)^{-1 / 2} \\
& \quad \simeq|x|^{2\left(h_{K_{3}+K_{4}}-h_{K_{1}}-h_{K_{2}}\right)} \\
& \quad \times \varkappa^{2} C_{K_{1}, K_{2}, K_{3}+K_{4}} C_{N-K_{3}, N-K_{4}, K_{3}+K_{4}}, \quad|x| \rightarrow 0 .
\end{aligned}
$$

It follows that (7.26) in fact gives us the correct expressions for the quantum part of the structure constants up to the overall constant multiplier $x$ independent on $K_{i}$. To find $x$ one has to consider the case $K_{1}+K_{2}=N$, and to extract the contribution of the identity operator to the OPE of $\sigma_{K_{1}}(0) \sigma_{K_{2}}(x)$.

But there is some difficulty involved. For $K_{1}+K_{2}=N$ the integrand in (7.28) has the logarithmic singularity for $|x| \rightarrow 0$ :

$$
Z_{*}\left(K_{1}, N-K_{1}, K_{3}, N-K_{3} \mid x\right) \simeq 2 \pi\left(\ln \frac{\gamma}{|x|}\right)|x|^{2 K_{1}\left(N-K_{1}\right) / N^{2}},
$$

where the constant $\gamma$ depends on $K_{1}, K_{3}$ and is equal,

$$
\begin{gathered}
\gamma\left(K_{1}, K_{3}\right)=\left[\delta\left(K_{1}\right) \delta\left(K_{3}\right)\right]^{1 / 2}, \\
\ln \delta(K)=2 \Psi(1)-\Psi(K / N)-\Psi(1-K / N) .
\end{gathered}
$$

The function $Z(x)$ has the same asymptotic behaviour and for the correlation function (7.29) we obtain 


$$
\begin{aligned}
& \left\langle\sigma_{K_{1}}(0) \sigma_{N-K_{1}}(x) \sigma_{K_{3}}(1) \sigma_{N-K_{3}}(\infty)\right\rangle_{\mathrm{qu}} \\
& \quad \simeq|x|^{-4 h_{K_{1}} \varkappa^{2}}\left[2 \pi \ln \frac{\gamma\left(K_{1}, K_{3}\right)}{|x|}\right]^{-1}, \quad|x| \rightarrow 0 .
\end{aligned}
$$

The appearance of the logarithmic singularity in this expression is connected with the fact that in the limit of an infinite radius of the orbifold the spectrum of conformal dimensions of fields in the OPE of $\sigma_{K}(0) \sigma_{N-K}(x)$ become continuous. Indeed, the operators $\mathcal{O}_{\sigma}$ present in the expansion

$$
\sigma_{N-K}(x) \sigma_{K}(0)=\sum_{\mathbf{p} \in \Lambda^{*}} C_{K}(\mathbf{p})|x|^{\boldsymbol{\sigma}^{2}-4 h_{K}}\left(\mathcal{O}_{\mathbf{\sigma}}(0)+\ldots\right)
$$

correspond to ordinary vertices

$$
\mathcal{O}_{\mathbf{p}}=\operatorname{expip}_{\mu} X^{\mu}, \quad \mu=1,2, X^{1}=X+X^{*}, \quad X^{2}=i\left(X^{*}-X\right)
$$

with dimension

$$
\Delta(\mathbf{p})=\mathbf{P}^{2} / 2
$$

The momentum $\mathbf{P}$ is taking values on the dual orbifold lattice $\Lambda^{*}$ with the volume of the fundamental cell

$$
V_{A}^{-1} \equiv V_{A^{*}} \sim R^{-2}
$$

where $R$ is the orbifold radius. It is also supposed in (7.33) that the operators are normalized as follows:

$$
\left\langle\mathcal{O}_{\mathbf{p}}(0) \mathcal{O}_{\mathbf{p}}(x)\right\rangle=|x|^{-2 \mathbf{p}^{2}}
$$

In the limit $R \rightarrow \infty$ the sum in (7.33) can be replaced by the integral, and under the choice

$$
C_{K}(\mathbf{p})=C_{N-K}(\mathbf{p})=\varkappa\left(2 \pi^{2} V_{\Lambda}\right)^{-1 / 2}[\delta(K)]^{-\mathbf{p}^{2} / 2}
$$

we obtain for the four-point function the following asymptotic

$$
\begin{aligned}
& \left\langle\sigma_{K_{1}}(0) \sigma_{N-K_{1}}(x) \sigma_{K_{3}}(1) \sigma_{N-K_{3}}(\infty)\right\rangle_{\mathrm{qu}} \\
& \quad \simeq \int V_{A} d^{2} \mathbf{p} C_{K_{1}}(\mathbf{p}) C_{K_{3}}(-\mathbf{p})|x|^{\mathbf{p}^{2}-4 h_{K_{1}}} \\
& \quad=|x|^{-4 h_{K_{1}} \chi^{2}\left[2 \pi \ln \left(\left[\delta\left(K_{1}\right) \delta\left(K_{3}\right)\right]^{1 / 2} /|x|\right)\right]^{-1}, \quad|x| \rightarrow 0,}
\end{aligned}
$$

coinciding with (7.32).

On the whole, the asymptotic (7.32) corresponds to the structure constants (7.36) of the algebra of operators $\sigma_{K}$ and $\mathcal{O}_{\mathbf{p}}$. Since $\mathcal{O}_{\mathbf{p}=0}$ is the identity operator, it follows from (7.36) that

$$
\left\langle\sigma_{K}(0) \sigma_{N-K}(x)\right\rangle=|x|^{-4 h_{K}} \chi\left(2 \pi^{2} V_{\Lambda}\right)^{-1 / 2}
$$

Thus to achieve the standard normalization of the two-point functions one has to put

$$
x=\left(2 \pi^{2} V_{\Lambda}\right)^{1 / 2}
$$


The resulting expressions for the structure constants are the following:

$$
\begin{gathered}
C_{K}^{\mathrm{qu}}(\mathbf{p})=[\delta(K)]^{-\mathbf{p}^{2} / 2}, \\
C_{K_{1} K_{2} K_{3}}^{\mathrm{qu}}=\left(2 \pi V_{A}\right)^{1 / 2}\left(\prod_{i=1}^{3} \frac{\Gamma\left(K_{i} / N\right)}{\Gamma\left(1-K_{i} / N\right)}\right)^{1 / 2}, \quad K_{1}+K_{2}+K_{3}=2 N .
\end{gathered}
$$

It is easy to see that for $K_{1}=K_{2}=K$ the expression (7.41) coincides, up to the contribution of instantons, with the structure constant $C_{\sigma_{+} \sigma_{+} \sigma_{--}}$computed in [5] [formula (4.47)]:

$$
C_{K, K, 2(N-K)}^{\mathrm{qu}}=\left(V_{A}\left|\operatorname{tg} \frac{\pi K}{N}\right|\right)^{1 / 2} \frac{\Gamma^{2}(K / N)}{\Gamma(2 K / N-1)}, \quad K>N / 2 .
$$

We have to point out that the constants (7.40) were also first computed in [5]. It is also possible to check that for the case

$$
K_{1}=N-K_{2}=K_{3}=N-K_{4},
$$

(7.28), (7.22) reduce to the corresponding expression in [5] [formula (4.39)].

Thus the general formulae (7.22), (7.28), and (7.41) complete the relations obtained in [5] for particular cases (7.42), (7.43). Besides, the integral representation constructed above considerably simplifies all the calculations and provides an effective description of the quantum part of multitwist correlation functions (7.22) for $M>4$. Note also that the expressions derived above describe the full sums of the perturbation theory series for the $\sigma$-model on the world-sheet of the string, propagating in the orbifold background.

\section{Conclusions}

We find on the whole that analytic fields in two dimensions play an important role in the conformal quantum field theory and in the theory of strings. Each time the naive transition to the $1^{\text {st }}$ order formalism exists it can be settled rigorously and leads to considerable simplifications. For the partition functions and correlation functions it provides expressions with an explicitly revealed complex analytical structure. We think that the transition to the analytic fields in conformal quantum field theory is analogous to the transition from real to complex analytic coordinates in geometry. For the present this connection can be expressed even more clearly $[1,2,7,8,11,12]$ and no doubt deserves further study.

Note Added. After this research had been completed, I was informed that M. Bershadsky and A. Radul computed the determinants of Laplace operators $\Delta_{j}$ for $Z_{N}$-symmetric surfaces extending the method by Al. Zamolodchikov [4]. Our discussions in Sects. 4 and 5 also have some intersections with their paper [19].

\section{Appendix}

For the sake of completeness we bring here the basic integrals we have met in Sect. 7. To calculate them one has to transform the integral over the complex plane 
into the sum of products of contour integrals over $d z$ and $d \bar{z}$ around cuts.

$$
\begin{aligned}
I(\alpha, \beta)= & \int d^{2} z|z|^{2 \alpha}|1-z|^{2 \beta} \\
= & \pi \frac{\Gamma(1+\alpha) \Gamma(1+\beta) \Gamma(-1-\alpha-\beta)}{\Gamma(-\alpha) \Gamma(-\beta) \Gamma(2+\alpha+\beta)} \\
I(a, b, c, x)= & \int d^{2} z|z|^{2 a}|z-x|^{2 b}|z-1|^{2 c} \\
= & \frac{\sin \pi a \sin \pi b}{\sin \pi(a+b)}\left|\int_{0}^{x} z^{a}(z-x)^{b}(z-1)^{c} d z\right|^{2} \\
& +\frac{\sin \pi c \sin \pi(a+b+c)}{\sin \pi(a+b)}\left|\int_{1}^{\infty} z^{a}(z-x)^{b}(z-1)^{c} d z\right|^{2} \\
= & \pi \frac{\Gamma(a+1) \Gamma(b+1) \Gamma(-a-b-1)}{\Gamma(-a) \Gamma(-b) \Gamma(a+b+2)}|x|^{2(a+b+1)} \\
& \times|F(a+1,-c, a+b+2, x)|^{2} \\
& +\pi \frac{\Gamma(a+b+1) \Gamma(c+1) \Gamma(-a-b-c-1)}{\Gamma(-a-b) \Gamma(-c) \Gamma(a+b+c+2)} \\
& \times|F(-a-b-c-1,-b,-a-b, x)|^{2}
\end{aligned}
$$

where $F$ denotes the hypergeometric function:

$$
F(\alpha, \beta, \gamma, z)=\frac{\Gamma(\gamma)}{\Gamma(\alpha) \Gamma(\gamma-\alpha)} \int_{0}^{1} t^{\alpha-1}(1-t)^{\gamma-\alpha-1}(1-z t)^{-\beta} d t
$$

\section{References}

1. Alvarez-Gaumé, L., Moore, G., Nelson, P., Vafa, C., Bost, J.B.: Bosonization in arbitrary genus. Phys. Lett. 178 B, 41-47 (1986)

2. Knizhnik, V.G.: Analytic fields on Riemann surfaces. Phys. Lett. 180 B, 247-254 (1986)

3. Dotsenko, Vl.S., Fateev, V.A.: Conformal algebra and multipoint correlation functions in $2 D$ statistical models. Nucl. Phys. B 240 [FS 12], 312-360 (1984);

Four-point correlation functions and the operator algebra in the $2 D$ conformal invariant theories with the central charge $C<1$. Nucl. Phys. B 251 [FS 13], 691-742 (1985)

4. Zamolodchikov, Al.B.: Correlation functions of spin operators in the Ashkin-Teller model and the scalar field on a hyperelliptic surface. Preprint. Moscow. 1985; ZhETF 90, 1808-1818 (1986)

5. Dixon, L., Friedan, D., Martinec, E., Shenker, S.: The conformal field theory of orbifolds. Preprint EFI 86-42

6. Belavin, A.A., Polyakov, A.M., Zamolodchikov, A.B.: Infinite conformal symmetry in twodimensional quantum field theory. Nucl. Phys. B 241, 333-380 (1984)

7. Belavin, A.A., Knizhnik, V.G.: Algebraic geometry and the geometry of quantum strings. Phys. Lett. 168 B, 201-206 (1986)

8. Belavin, A.A., Knizhnik, V.G.: Complex geometry and theory of quantum strings. ZhETF 91, 364-390 (1986); Landau Inst. Preprint -9- 1986

9. Polyakov, A.M.: Quantum geometry of bosonic string. Phys. Lett. 103 B, 207-210 (1981)

10. Fay, J.D.: Theta functions on Riemann surfaces. In: Lecture Notes in Mathematics, Vol. 352. Berlin, Heidelberg, New York: Springer 1975

11. Alvarez-Gaumé, L., Moore, G., Vafa, C.: Theta-functions, modular invariance and strings. Commun. Math. Phys. 106, 40-87 (1986) 
12. Beilinson, A.A., Manin, Yu.I.: The Mumford form and the Polyakov measure in string theory. Commun. Math. Phys. 107, 359-376 (1986)

13. Belavin, A.A., Knizhnik, V.G., Morozov, A.Yu., Perelomov, A.M.: Two- and three-loop amplitudes in the bosonic string theory. Phys. Lett. 177 B, 324-328 (1986)

14. Moore, G.: Modular forms and two-loop string physics. Phys. Lett. 176 B, 69-73 (1986)

15. Gliozzi, F., Olive, D., Sherk, J.: Supersymmetry, supergravity theories and the dual spinor model. Nucl. Phys. B 122, 253-290 (1977)

16. Schwarz, J.H.: Superstrings. Phys. Rep. 89 C, 223 (1982)

17. Bost, J.B., Jolicoeur, T.: A holomorphy property and critical dimension in string theory from an index theorem. Phys. Lett. 174 B, 273-278 (1986)

18. Bismut, J.-M., Freed, D.S.: The analysis of elliptic families. I, II. Commun. Math. Phys. 106, 159-176 (1986) and 107, 103-163 (1986)

19. Bershadsky, M., Radul, A.: Conformal field theories with additional $Z_{N}$ symmetry. Preprint. Moscow. - 1986

Communicated by Ya. G. Sinai

Received March 1, 1987 